\title{
Temporal Genetic Structure of Phytophthora capsici Populations from a Creek Used for Irrigation in Michigan
}

L. M. Quesada-Ocampo and L. L. Granke, Visiting Research Associates, and M. K. Hausbeck, Professor, Department of Plant Pathology, Michigan State University, East Lansing, MI 48824

\begin{abstract}
Quesada-Ocampo, L. M., Granke, L. L., and Hausbeck, M. K. 2011. Temporal genetic structure of Phytophthora capsici populations from a creek used for irrigation in Michigan. Plant Dis. 95:1358-1369.

Irrigation water may harbor Phytophthora capsici, and irrigating susceptible vegetable crops with infested water can initiate epidemics. In this study, we evaluated the genetic structure of 106 P. capsici isolates collected from a creek used for irrigation $(2002,2003$, and 2006) and from a field adjacent to the creek (2001) using six polymorphic nuclear loci. Bayesian clustering analysis detected four clusters in the sample, and some clusters occurred more or less frequently in certain years. In 2006, isolates belonging to cluster four predominated in the sampling. Mean pairwise $\mathrm{F}_{\mathrm{ST}}$ values (0.008 to 0.065 ) indicated low differentiation

2006 was compared to 2001 and 2002. Differences in isolate phenotypic traits were observed year-to-year. Isolates insensitive to mefenoxam were more common in 2006 and 2003 than in 2002 and 2001. The mating type ratio was approximately $1: 1$ in 2002 and 2003, but was skewed toward A1 in 2001 and toward A2 in 2006. Since irrigation water can remain infested or become reinfested annually with $P$. capsici for years after the adjacent fields are transitioned to nonsusceptible crops, growers are advised to avoid potentially infested irrigation water even after rotating to nonhost crops for several years.
\end{abstract} between categories, but the most differentiation was observed when
Infested irrigation water has long been recognized as an important source of inocula for fungal and oomycete plant pathogens including Pythium spp. and Phytophthora spp. (3,10,41). Phytophthora capsici Leonian is a destructive soilborne pathogen that can infect economically important vegetable crops in the Solanaceae, Cucurbitaceae, and Fabaceae (5). Water is key for dispersal of $P$. capsici within a field (40) and potentially between fields if infested irrigation water is used (9). Previous research suggests that $P$. capsici does not overwinter and persist in surface water (9) and that irrigation water must become infested each growing season. Runoff water from infested fields can transport $P$. capsici from diseased plants to adjacent irrigation water sources (13). Filtration (3), ultraviolet irradiation (8), ozonation, chlorination (15), surfactants (45), and algaecides (12) may be used to disinfest irrigation water containing oomycete plant pathogen propagules.

Information on the occurrence, distribution, and population dynamics of $P$. capsici in irrigation water $(9,47)$ is essential to supplement the data available for field populations (24-29) and formulate robust disease management strategies (10). Genetic fingerprinting of $P$. capsici isolates from various surface water irrigation sources (river, creek, pond, ditch) in Michigan using amplified fragment length polymorphisms (AFLP) showed no association between AFLP similarity groups and specific locations or years (9). However, the populations used in this study were small $(<15$ isolates/site-year), so analyses that include more isolates could provide further information about the distribution of genetic variation of $P$. capsici from surface water irrigation sources in space and time. When the population variation of $P$. capsici isolates from irrigation ponds in Georgia was analyzed using simple sequence repeat markers (SSRs), low genetic diversity was found within

Corresponding author: M. K. Hausbeck, E-mail: hausbec1@msu.edu

L. M. Quesada-Ocampo and L. L. Granke made equal contributions to this work.

Accepted for publication 20 May 2011.

doi:10.1094/PDIS-03-11-0191

(c) 2011 The American Phytopathological Society each pond, but high diversity was found between ponds (47). Both of these studies used distance-based phylogenetic methods to cluster genetically similar individuals, providing important information about the genetic diversity of isolates within and between populations (36). Nonetheless, population genetics may be used to determine admixture between individual isolates and to separate subpopulations at a finer scale. In addition, $P$. capsici was frequently detected in a creek used for irrigation after the adjacent field was planted to potatoes (9). Previous work has not investigated changes in the temporal genetic structure of $P$. capsici isolates from irrigation water when the fields adjacent to the water were transitioned to nonhost production for several years. The objective of this study was to determine the genetic structure of $P$. capsici populations from a creek in Michigan that is used for irrigation. Specifically, we sought to determine whether $P$. capsici isolates from a single irrigation source show significant temporal population structure and genetic differentiation, and the distribution of genotypes from each genetic cluster when isolates were grouped by mating type, mefenoxam resistance, year, and source of isolation (irrigation water or field). Some information about 57 of the 106 isolates used in this study has been previously reported (9).

\section{Materials and Methods}

Isolate selection, maintenance, phenotypic characterization, and DNA extraction. A total of $91 P$. capsici isolates originating from a creek used for irrigation on a farm in Southwest Michigan and an additional 15 isolates from a field adjacent to the creek were obtained from the culture collection maintained in the laboratory of M. K. Hausbeck at Michigan State University (MSU) (Table 1). Isolates from 2002 to 2006 had been previously baited from the creek using cucurbit fruits and placed into long-term storage as described in the paper by Gevens et al. (9), including those isolates that were not included in the Gevens et al. study. The creek is less than a mile long and flows into a larger river. The sampling location was directly under a cattle bridge in a pasture area on the farm, near the mouth of the creek. In 2001, cucumbers showing symptoms of Phytophthora rot and/or pathogen signs were collected from a field adjacent to the creek. These cucumbers were transported to the Hausbeck Lab in a cooler, and tissue sections from the margin of lesions were plated onto unclarified V8 agar (UCV8A; $3 \mathrm{~g} \mathrm{CaCO}_{3}$, $15 \mathrm{~g}$ agar, $160 \mathrm{ml}$ unfiltered $\mathrm{V} 8$ juice, and $840 \mathrm{ml}$ distilled water) 
plates amended with $25 \mathrm{ppm}$ of benomyl, $100 \mathrm{ppm}$ of ampicillin, 30 ppm of rifampicin, and $100 \mathrm{ppm}$ of pentachloronitrobenzene (BARP). Plates were incubated at room temperature under continuous fluorescent lighting for 3 to 5 days. The resulting colonies were observed using a compound microscope, and $P$. capsici was identified using morphological characteristics according to the Phytophthora spp. key by Waterhouse (48). Resulting singlezoospore (24) isolates were placed into long-term storage (two 8-mm agar plugs were stored at $20^{\circ} \mathrm{C}$ in sterile $1.5-\mathrm{ml}$ microcentrifuge tubes with $1 \mathrm{ml}$ of sterile water and two sterile hemp seeds).

Isolates were retrieved from long-term stock cultures and grown on UCV8A as previously described (37). Morphological characteristics were used according to the Phytophthora spp. key by Waterhouse (48) to confirm the isolates were P. capsici, and the mating type (MT) and mefenoxam sensitivity of each isolate was determined as described previously (24). In addition, each isolate was tested for pathogenicity to pepper fruit (Capsicum annuum). Pepper fruits were surface disinfested in a $10 \%$ sodium hypochlorite solution, rinsed with distilled water, air-dried, and wounded on each side of the fruit using a sterile 1-mm dissecting needle. One fruit was used for each isolate. A 6-mm-diameter agar plug from an actively growing culture was placed over each wound and covered using a 12-mm-diameter microcentrifuge tube screw cap, which was affixed to the fruit surface with petroleum jelly. Fruits were visually checked for disease symptoms and pathogen signs after 4 days incubation in a humid chamber at room temperature $(25 \pm$ $\left.2^{\circ} \mathrm{C}\right)$. The experiment was conducted twice.

To obtain agar-free tissue for DNA extraction, agar plugs from an actively growing culture of each isolate were placed into $50-\mathrm{ml}$ centrifuge tubes containing $30 \mathrm{ml}$ of unclarified V8 juice broth (UCV8B; $3 \mathrm{~g} \mathrm{CaCO}_{3}, 160 \mathrm{ml}$ unfiltered V8 juice, and $840 \mathrm{ml}$ distilled water) and incubated at room temperature $\left(25 \pm 2^{\circ} \mathrm{C}\right)$ under constant fluorescent lighting on a shaker $(0.03 \times g) 2$ to 5 days or until pathogen growth was apparent. DNA was extracted from between 0.1 and $1 \mathrm{~g}$ of ground tissue using the DNeasy Plant Minikit (Qiagen) according to the manufacturer's protocol. A NanoDrop ND 1000 spectrophotometer and NanoDrop 2.4.7c software (NanoDrop Technologies Inc.) were used for quantification of genomic DNA.

PCR amplification and DNA sequencing. PCR amplification and DNA sequencing was accomplished according to the methods of Quesada-Ocampo et al. and using the same primers (38). Briefly, four regions of the mitochondrial genome (Cox1, Cox2, Nad1, and Nad5) and six nuclear genes ( $\beta$-tubulin, EF-1 $\alpha$, Enolase, HSP90, TigA, and Ura3) were amplified by polymerase chain reaction (PCR) (Table 2); PCR products were cleaned using ExoSAP-IT (Affymetrix, Inc.) and sent to the Michigan State University Research Technology Support Facility (East Lansing, MI) for sequencing. An ABI PRISM 3100 Genetic Analyzer (Applied Biosystems) was used to conduct two cycle sequencing reactions ( $1 \mu \mathrm{l}$ purified PCR product, $3 \mu \mathrm{l}$ primer, $8 \mu$ l sterile water) on the clean PCR product. Since a concurrent study with $P$. capsici using the same primers that were used in this study found that PHASE (46) as implemented in DnaSPv5 (30) was able to resolve haplotypes with a high degree of accuracy (38), the haplotypes of most samples with heterozygote positions were inferred using PHASE. The pGEM-T Easy Vector System (Promega) and Subcloning Efficiency DH5 $\alpha$ Competent Cells (Invitrogen) were used according to the manufacturer's instructions to clone the corresponding PCR product of a subset of samples with heterozygote positions (20\%), as determined from sequence analysis. The QIAquick PCR purification kit (Qiagen) was used to purify cloned PCR products according to the manufacturer's instructions. The purified products were amplified and sequenced as described above. Obtained haplotypes were confirmed by using PHASE (46) as implemented in DnaSPv5 (30) by running simulations with 5,000 iterations. In all cases, the inferred haplotype with cloning and direct sequencing data matched the haplotypes obtained using PHASE. Haplotypes of remaining samples with heterozygote positions were inferred with PHASE.
Sequence analysis. Lasergene SeqMan Pro version 8.0 (DNASTAR Inc.) was used to manually edit base calls and sequence alignment. MacClade (31) was used to convert FASTA files from Lasergene SeqMan Pro into NEXUS files for subsequent analyses. To confirm that the sequences obtained corresponded to the $P$. capsici target genes, our data were compared to publicly available sequence data via nucleotide BLAST (1).

Data analyses. Sequences from mitochondrial and nuclear loci were analyzed individually. DnaSPv5 (30) was used to collapse sequence data into unique haplotypes and to classify base substitutions as phylogenetically informative or uninformative, transitions or transversions, and synonymous or nonsynonymous substitutions. Genetic diversity, neutrality, recombination, and genetic differentiation (Wright's $\mathrm{F}_{\mathrm{ST}}$ ) were estimated using DnaSPv5 for the entire collection of isolates and for isolates grouped by predefined categories of year, source (field versus water), mating type, and mefenoxam sensitivity. The significance of genetic differentiation indexes was determined from 5,000 random permutations. The program TCS Ver. 1.13 (4) allowed for the creation of statisticalparsimony genealogies for haplotypes. SplitsTree Ver. $4 \mathrm{~b} 06$ (18) was used to generate NeighborNet networks based on uncorrected $\mathrm{P}$ distances for each locus using the split network method with 10,000 bootstrap replicates to assess support.

The assignment of individuals to genetic clusters was carried out using a Bayesian clustering algorithm implemented in the program Structure 2.3X (36). All loci were individually coded as haplotypes, and an admixture model was used to estimate the number of genetic clusters $(\mathrm{K})$. The optimal number of $\mathrm{K}$ was determined by comparing posterior distribution likelihoods among three independent runs of $\mathrm{K}=1$ to 20 . After a burn-in period of 200,000 iterations, runs of 100,000 Markov chain Monte Carlo (MCMC) iterations with correlated allele frequencies were carried out (lambda =1). No prior population information was assumed for the analyses. However, population structure figures with prior population information and sorted by the proportional membership (Q) of an isolate in a cluster were generated using the Population Sorting Tool (PST), a graphic editing R (39) program (J. J. Morrice, unpublished data). Isolates were assigned to year, mating type, and mefenoxam sensitivity categories for figure generation.

Nucleotide sequence accession numbers. Sequences for each isolate reported in this paper have been deposited in the GenBank database as the following accessions: HQ725098 to HQ726793.

\section{Results}

No polymorphisms were observed in mitochondrial sequence data (Table 3), and these genes were excluded from all analyses. Sequence data from the six nuclear loci revealed 49 polymorphisms, 46 of which were parsimony informative (Table 3). Most of the nucleotide substitutions were synonymous (37 of 49); both transitions (40 of 49) and transversions (9 of 49) were detected. Two allelic variants were observed for all polymorphic sites (Table 3 ). The highest numbers of polymorphisms were found in Enolase $(s=16)$ and Ura3 $(s=11)$ (Table 4). The percentage of polymorphism in the other nuclear genes sequenced ranged from 1 to $2 \%$, whereas $3 \%$ of the Enolase and Ura3 nucleotides sequenced were polymorphic. Estimates of nucleotide diversity $(\pi)$ suggest that Ura3 contains the most nucleotide diversity of the genes sequenced followed by EF-1 $\alpha$ and Enolase, respectively. Watterson's theta estimators for the genes also supported Enolase and Ura3 as the most diverse. Haplotype diversity (Hd) estimates suggest that Enolase contained the highest level of DNA polymorphism, followed by Ura3 and EF-1 $\alpha$ (Table 4). Between 4 and 24 haplotypes were detected for all of the nuclear genes (Table 1). Unique haplotypes occurred in all of the genes except TigA and constituted 7\% (EF-1 $\alpha$ ) to $40 \%$ (HSP90) of the haplotypes detected depending on the gene. In total, 93 multilocus genotypes were found among the 106 isolates collected (Table 1). Three of these multilocus genotypes included isolates of both A1 and A2 mating type, for a total of 96 multilocus genotypes if mating type is taken into account. A single A1 isolate from 2001 (13127) shared a multilocus genotype 
Table 1. Isolates of Phytophthora capsici used in this study

\begin{tabular}{|c|c|c|c|c|c|c|c|c|}
\hline Isolate code & Month & Year & Source $^{a}$ & Bait $^{\text {b }}$ & $\mathbf{M T}^{\mathbf{c}}$ & $\mathbf{M S}^{\mathbf{d}}$ & $\mathrm{GC}^{\mathrm{e}}$ & Haplotypes $^{\mathrm{f}}$ \\
\hline 7194 & Sep. & 2001 & $\mathrm{~F}$ & Csa & A1 & $\mathrm{S}$ & 3 & $1-1,7-1,2-1-1,2-2,3$ \\
\hline 7195 & Sep. & 2001 & $\mathrm{~F}$ & Csa & A1 & $\mathrm{S}$ & 1 & $1-1,5-1,2-1-1-3$ \\
\hline 7196 & Sep. & 2001 & $\mathrm{~F}$ & Csa & $\mathrm{A} 2$ & $\mathrm{~S}$ & 1 & $1,3-1,6-1,2-1-1,3-3$ \\
\hline 7197 & Sep. & 2001 & $\mathrm{~F}$ & Csa & $\mathrm{A} 2$ & $\mathrm{~S}$ & 4 & $1,3-1,10-1,4-1-1-2,3$ \\
\hline 7198 & Sep. & 2001 & $\mathrm{~F}$ & Csa & $\mathrm{A} 2$ & $\mathrm{~S}$ & 2 & $10-1,8-3,7-1,3-2,3-20$ \\
\hline 7199 & Sep. & 2001 & $\mathrm{~F}$ & Csa & A1 & $\mathrm{I}$ & 2 & $10-1,8-3,7-1,3-2,3-20$ \\
\hline 7200 & Sep. & 2001 & $\mathrm{~F}$ & Csa & A1 & $\mathrm{S}$ & 1 & $1,3-1,4-1,4-1-1-3$ \\
\hline 7201 & Sep. & 2001 & $\mathrm{~F}$ & Csa & A1 & $\mathrm{S}$ & 1 & $1,2-1,4-1,4-1-1,3-2,3$ \\
\hline 7203 & Sep. & 2001 & $\mathrm{~F}$ & Csa & A2 & $\mathrm{S}$ & 2 & $1,2-1,4-1,4-1-1,2-21,22$ \\
\hline 7206 & Sep. & 2001 & $\mathrm{~F}$ & Csa & $\mathrm{A} 2$ & $\mathrm{~S}$ & 2 & $1,2-1,4-1,2-1-1,2-13,14$ \\
\hline 7208 & Sep. & 2001 & $\mathrm{~F}$ & Csa & A1 & $\mathrm{S}$ & 2 & $1,2-1,4-1,2-1-1,2-15,16$ \\
\hline 7210 & Sep. & 2001 & $\mathrm{~F}$ & Csa & A1 & $\mathrm{S}$ & 2 & $1,2-1,3-2,4-1-1,3-3,17$ \\
\hline 9533 & Sep. & 2002 & $\mathrm{~W}$ & Csa & A2 & $\mathrm{I}$ & 1 & $1-1,7-1,4-1-1-3,4$ \\
\hline 9537 & Sep. & 2002 & $\mathrm{~W}$ & Csa & A2 & IS & 2 & $4-1,4-1,4-1-1-3,4$ \\
\hline 9546 & Aug. & 2002 & $\mathrm{~W}$ & Csa & A1 & IS & 1 & $1,2-1,6-1,4-1-1-2,5$ \\
\hline 9547 & Sep. & 2002 & W & Csa & $\mathrm{A} 2$ & IS & 2 & $1,5-1,5-2-1-1-3,6$ \\
\hline 9550 & Aug. & 2002 & W & Pco & A1 & IS & 2 & $1,2-1,12-2,5-1-1-5,6$ \\
\hline 9553 & Aug. & 2002 & W & Csa & A2 & $\mathrm{I}$ & 1 & $1-1,6-1,2-1-1,2-5,6$ \\
\hline 9554 & Aug. & 2002 & W & Csa & A1 & IS & 1 & $1,2-1,6-1,4-1-1-5,6$ \\
\hline 9556 & Aug. & 2002 & W & Csa & A1 & $\mathrm{I}$ & 2 & $1-1,3-4,6-1-1-7,8$ \\
\hline 9558 & Sep. & 2002 & W & Csa & $\mathrm{A} 2$ & I & 1,3 & $1-1,7-1,4-1-1-3$ \\
\hline 9559 & Aug. & 2002 & $\mathrm{~W}$ & Csa & $\mathrm{A} 2$ & I & 3 & $1,2-1,7-1,4-1-1-2,3$ \\
\hline 9561 & Sep. & 2002 & W & Csa & A2 & IS & 3 & $1,2-1,7-1,2-1-1-2,3$ \\
\hline 9565 & Sep. & 2002 & $\mathrm{~W}$ & Csa & A1 & $\mathrm{S}$ & 1 & $1-1,2-1,4-1,3-1,3-3$ \\
\hline 9569 & Aug. & 2002 & $\mathrm{~W}$ & Csa & A2 & IS & 2 & $1,2-1,4-1,2-1,3-1,3-2,9$ \\
\hline 9570 & Aug. & 2002 & W & Csa & A1 & $\mathrm{I}$ & 1 & $1,2-1,6-3,4-1-1-2,5$ \\
\hline 9571 & Aug. & 2002 & W & Pco & A1 & $\mathrm{I}$ & 2 & $1-1,3-4,6-1-1-3,10$ \\
\hline 9572 & Aug. & 2002 & W & Csa & A1 & IS & 2 & $1,2-1,12-4,6-1-1-2,5$ \\
\hline 9577 & Aug. & 2002 & $\mathrm{~W}$ & Csa & A1 & IS & 1 & $1-1,4-3,4-1,3-1-5$ \\
\hline 9580 & Aug. & 2002 & $\mathrm{~W}$ & Csa & A2 & $\mathrm{I}$ & 1 & $1-1,4-1,4-1-1,3-2,5$ \\
\hline 9581 & Aug. & 2002 & W & Csa & A1 & $\mathrm{S}$ & 1 & $1,2-1,6-1,4-1-1-2,5$ \\
\hline 9583 & Sep. & 2002 & W & Csa & $\mathrm{A} 2$ & $\mathrm{~S}$ & 3 & $1,2-1,7-1,5-1-1,3-2$ \\
\hline 9586 & Sep. & 2002 & W & Csa & A2 & IS & 3 & $1,2-1,3-1,2-1-1,4-2,3$ \\
\hline 9588 & Sep. & 2002 & $\mathrm{~W}$ & Csa & A1 & $\mathrm{S}$ & 1 & $1-1,2-4-1,3-1,4-3$ \\
\hline 9598 & Sep. & 2002 & $\mathrm{~W}$ & Csa & A1 & $\mathrm{S}$ & 1 & $1-1,2-4-1,3-1,4-3$ \\
\hline 9642 & Aug. & 2003 & $\mathrm{~W}$ & Csa & $\mathrm{A} 2$ & $\mathrm{~S}$ & 4 & $1,2-1,10-2,4-1-1-2,11$ \\
\hline 9643 & Aug. & 2003 & W & Csa & A1 & $\mathrm{I}$ & 4 & $1,2-1,10-2,4-1,4-1-1,2$ \\
\hline 9644 & Jul. & 2003 & W & Pco & A1 & $\mathrm{I}$ & 1 & $1,6-1,4-1,4-1-1-2,3$ \\
\hline 9649 & Jul. & 2003 & $\mathrm{~W}$ & Csa & A1 & $\mathrm{I}$ & 1 & $1,2-1,2-3,4-1-1-5$ \\
\hline 9650 & Aug. & 2003 & W & Csa & A1 & I & 1 & $1-1,2-3,4-1-1,4-2$ \\
\hline 9652 & Aug. & 2003 & $\mathrm{~W}$ & Csa & A1 & $\mathrm{I}$ & 3 & $1,2-1,11-1,2-1-1-2,3$ \\
\hline 9655 & Aug. & 2003 & W & Csa & A2 & $\mathrm{S}$ & 3 & $1,4-1,11-1,2-1-1,2-2,3$ \\
\hline 9669 & Aug. & 2003 & W & Csa & $\mathrm{A} 2$ & IS & 1 & $1-1,4-3,4-1-1-3$ \\
\hline 9670 & Jul. & 2003 & $\mathrm{~W}$ & Csa & A1 & $\mathrm{I}$ & 2 & $1-1,4-20,21-1-1-3,5$ \\
\hline 9672 & Aug. & 2003 & $\mathrm{~W}$ & Csa & A1 & I & 2,3 & $1,2-1,13-1,3-1-1-2,3$ \\
\hline 9673 & Aug. & 2003 & W & Pco & A2 & $\mathrm{I}$ & 3 & $1,2-1,2-2,5-1-1,3-2$ \\
\hline 9677 & Aug. & 2003 & W & Csa & A1 & $\mathrm{I}$ & 2 & $3,5-1,5-1,4-1-1,4-3,12$ \\
\hline 9680 & Aug. & 2003 & W & Pco & $\mathrm{A} 2$ & $\mathrm{I}$ & 3 & $1,2-1,2-1,5-1,5-1,3-2$ \\
\hline 9683 & Aug. & 2003 & W & Csa & A2 & IS & 3 & $1,2-1,2-1,10-1-1,3-2$ \\
\hline 9685 & Aug. & 2003 & W & Pco & A2 & $\mathrm{I}$ & 3 & $1,2-1,2-1,5-1-1,3-2$ \\
\hline 9687 & Aug. & 2003 & W & Csa & A1 & $\mathrm{I}$ & 1 & $1,2-1,2-1-1-1-3,5$ \\
\hline 9688 & Aug. & 2003 & W & Csa & $\mathrm{A} 2$ & IS & 3 & $1,2-1,2-1,5-1-1,3-2$ \\
\hline 9691 & Jul. & 2003 & W & Csa & A1 & $\mathrm{I}$ & 1 & $1-1,4-1,4-1-1-3,5$ \\
\hline 9693 & Aug. & 2003 & W & Pco & $\mathrm{A} 2$ & $\mathrm{I}$ & 2 & $1,2-1,4-1,10-1-1-2,9$ \\
\hline 9697 & Aug. & 2003 & $\mathrm{~W}$ & Csa & A1 & $\mathrm{I}$ & 2,3 & $1,2-1,9-1,3-1-1,3-2,3$ \\
\hline 9698 & Jul. & 2003 & $\mathrm{~W}$ & Csa & A1 & I & 1 & $1,2-1,4-1,4-1-1-3,5$ \\
\hline 9726 & Jul. & 2003 & $\mathrm{~W}$ & Csa & A1 & $\mathrm{I}$ & 2 & $1-1,4-15,16-1-1-3,5$ \\
\hline 9733 & Jul. & 2003 & W & Csa & A1 & IS & 1 & $1-1,12-1,4-1-2,3-3,5$ \\
\hline 10037 & Sep. & 2003 & W & Pco & $\mathrm{A} 2$ & $\mathrm{~S}$ & 3 & $1-1,2-1,2-1-1-2$ \\
\hline 12098 & Jul. & 2006 & W & Cpe & $\mathrm{A} 2$ & $\mathrm{I}$ & 3 & $2,7-1,5-1,4-1-1-2,11$ \\
\hline 12099 & Jul. & 2006 & $\mathrm{~W}$ & Cpe & A2 & $\mathrm{I}$ & 4 & $1,2-1,5-3,4-1-1-1,2$ \\
\hline 12100 & Jul. & 2006 & W & Cpe & A2 & $\mathrm{I}$ & 4 & $2,7-1,5-1,4-1-1-1,2$ \\
\hline \multirow[t]{2}{*}{12101} & Jul. & 2006 & W & Cpe & $\mathrm{A} 2$ & IS & 3 & $2,8-1,14-1,4-1-1-2,3$ \\
\hline & & & & & & & & (continued on nex \\
\hline
\end{tabular}

a Isolates were either baited from irrigation water $(\mathrm{W})$ or isolated from cucumbers from a field adjacent to the water source (F) in southwest Michigan.

b Isolates were retrieved from irrigation water using cucumber (Csa), pear (Pco), or zucchini/summer squash (Cpe) fruits as baits or were isolated from fieldgrown cucumber (Csa) fruits.

c Mating type (MT) (A1 or A2) of an isolate.

${ }^{\mathrm{d}}$ Mefenoxam Sensitivity (MS). Isolates grown on media amended with $100 \mathrm{ppm}$ mefenoxam were compared to an unamended control: Sensitive (S): $<30 \%$ relative growth; Intermediately Sensitive (IS): 30 to $90 \%$ relative growth; Insensitive (I): $>90 \%$ relative growth.

e Predominant genetic cluster(s) (GC). If an isolate doe not have $>0.1$ proportion membership higher in one genetic cluster than another, both clusters are listed.

${ }^{\mathrm{f}}$ Each number represents the haplotype number for both alleles (one before comma, one after comma) per gene in the following order: $\beta$ tub-EF-1 $\alpha$-EnolaseHSP90-TigA-Ura3. Genotype phase is unknown. For Cox1-Cox2-Nad1-Nad5, 1-1-1-1 may represent all isolates as no polymorphisms were found. 
Table 1. (continued from preceding page)

\begin{tabular}{|c|c|c|c|c|c|c|c|c|}
\hline Isolate code & Month & Year & Source $^{a}$ & Bait $^{b}$ & $\mathbf{M T}^{\mathrm{c}}$ & $\mathbf{M S}^{\mathbf{d}}$ & $\mathbf{G C}^{\mathrm{e}}$ & Haplotypes $^{\mathrm{f}}$ \\
\hline 12102 & Jul. & 2006 & $\mathrm{~W}$ & Cpe & A1 & $\mathrm{S}$ & 3 & $2,8-1,14-1,4-1-1-2,3$ \\
\hline 12103 & Jul. & 2006 & W & Cpe & $\mathrm{A} 2$ & IS & 3 & $2,8-1,7-1,2-1-1-2,3$ \\
\hline 12105 & Jul. & 2006 & W & Cpe & A1 & $\mathrm{S}$ & 3 & $1,3-1,11-5,18-1-1,2-2,3$ \\
\hline 12106 & Jul. & 2006 & W & Cpe & A2 & IS & 3,4 & $2,3-1,7-1,4-1-1-2$ \\
\hline 12107 & Jul. & 2006 & $\mathrm{~W}$ & Cpe & A2 & $\mathrm{S}$ & 1 & $1,3-1,5-4-1-3-3,5$ \\
\hline 12108 & Jul. & 2006 & W & Cpe & A2 & $\mathrm{S}$ & 1 & $1-1,5-1,5-1-1,3-3,5$ \\
\hline 12109 & Jul. & 2006 & W & Cpe & A2 & $\mathrm{S}$ & 1 & $1-1,5-4,14-1-1,3-3,5$ \\
\hline 12110 & Jul. & 2006 & W & Cpe & A1 & IS & 2 & $1-1,10-7,18-1-1,3-2,3$ \\
\hline 12111 & Jul. & 2006 & W & Cpe & A1 & $\mathrm{S}$ & 2 & $3,9-1,4-12,13-1-1-5,11$ \\
\hline 12112 & Jul. & 2006 & $\mathrm{~W}$ & Cpe & A2 & $\mathrm{S}$ & 1 & $1,3-1,5-4-1-1,3-3,5$ \\
\hline 12113 & Aug. & 2006 & W & Csa & $\mathrm{A} 2$ & I & 4 & $1,3-1,5-7,19-1-1-2,6$ \\
\hline 12114 & Aug. & 2006 & W & Csa & A1 & $\mathrm{I}$ & 4 & $2,3-1,5-8,9-1-1-2,11$ \\
\hline 12115 & Aug. & 2006 & W & Csa & A1 & I & 4 & $1,2-1,5-7,19-1-1-1,2$ \\
\hline 12116 & Aug. & 2006 & $\mathrm{~W}$ & Csa & A2 & $\mathrm{I}$ & 4 & $1,2-1,5-7,11-1-1-2,11$ \\
\hline 12118 & Aug. & 2006 & $\mathrm{~W}$ & Csa & A2 & I & 4 & $1,2-1,5-1,4-1-1-2,11$ \\
\hline 12119 & Aug. & 2006 & W & Csa & A2 & I & 4 & $1,2-1,5-1,4-1-1-2,11$ \\
\hline 12120 & Aug. & 2006 & W & Cpe & A2 & $\mathrm{I}$ & 4 & $1,2-1,5-1,4-1-1-2,11$ \\
\hline 12121 & Aug. & 2006 & W & Cpe & A2 & I & 4 & $2,3-1,5-1,14-1-1-2,11$ \\
\hline 12122 & Aug. & 2006 & W & Cpe & $\mathrm{A} 2$ & I & 4 & $2,3-1,5-1,14-1-1-2,11$ \\
\hline 12123 & Aug. & 2006 & W & Cpe & A2 & I & 4 & $1,2-1,5-1,14-1-1-2,11$ \\
\hline 12124 & Aug. & 2006 & W & Cpe & $\mathrm{A} 2$ & I & 4 & $1,2-1,5-1,2-1-1-2,11$ \\
\hline 12125 & Aug. & 2006 & W & Cpe & A 2 & $\mathrm{I}$ & 4 & $2,3-1,5-1,22-1-1-2,11$ \\
\hline 12126 & Aug. & 2006 & W & Cpe & A 2 & I & 4 & $2,3-1,5-7,18-1-1-1,2$ \\
\hline 12127 & Aug. & 2006 & W & Cpe & $\mathrm{A} 2$ & I & 4 & $2,3-1,5-12,18-1-1-2,11$ \\
\hline 12130 & Aug. & 2006 & $\mathrm{~W}$ & Cpe & A2 & $\mathrm{S}$ & 1 & $1-1,5-5,12-1-1,3-3,5$ \\
\hline 12131 & Aug. & 2006 & $\mathrm{~W}$ & Cpe & A2 & I & 4 & $1,2-1,5-5,23-1-1-2,11$ \\
\hline 12133 & Aug. & 2006 & $\mathrm{~W}$ & Cpe & A2 & $\mathrm{S}$ & 1 & $2,3-1,10-1,5-1-1,3-3,5$ \\
\hline 12134 & Aug. & 2006 & W & Cpe & A2 & $\mathrm{S}$ & 1 & $1-1,5-4,17-1-1,3-3,5$ \\
\hline 12136 & Aug. & 2006 & W & Cpe & A1 & $\mathrm{S}$ & 4 & $1,3-1,10-4,17-1-1,3-1,2$ \\
\hline 12137 & Aug. & 2006 & W & Cpe & A1 & IS & 4 & $1-1,10-2,18-1-1,3-1,2$ \\
\hline 12138 & Aug. & 2006 & W & Cpe & $\mathrm{A} 2$ & IS & 4 & $1-1,10-4,14-1-1,3-1,2$ \\
\hline 12139 & Aug. & 2006 & W & Cpe & $\mathrm{A} 2$ & I & 4 & $1,2-1,5-2,14-1-1-1,2$ \\
\hline 12140 & Aug. & 2006 & W & Cpe & A2 & I & 4 & $1,2-1,5-1,24-1-1-1,2$ \\
\hline 12141 & Aug. & 2006 & W & Cpe & A1 & I & 3 & $1-1,11-1,24-1-1-2$ \\
\hline 12142 & Aug. & 2006 & W & Cpe & $\mathrm{A} 2$ & I & 3 & $1-1,11-2,5-1-1-2$ \\
\hline 12144 & Aug. & 2006 & W & Csa & A1 & IS & 4 & $2,3-1,5-1,4-1-1-1,2$ \\
\hline 12146 & Aug. & 2006 & W & Csa & A2 & $\mathrm{I}$ & 4 & $2,3-1,5-1,4-1-1-1,2$ \\
\hline 12147 & Aug. & 2006 & W & Csa & $\mathrm{A} 2$ & I & 4 & $2,3-1,5-3-1,2-1-1,2$ \\
\hline 12148 & Aug. & 2006 & $\mathrm{~W}$ & Csa & $\mathrm{A} 2$ & $\mathrm{I}$ & 4 & $2,3-1,5-1,14-1-1-1,2$ \\
\hline 13127 & Aug. & 2001 & $\mathrm{~F}$ & Csa & A1 & $\mathrm{S}$ & 4 & $2,3-1,5-1,4-1-1-1,2$ \\
\hline 13131 & Aug. & 2001 & $\mathrm{~F}$ & Csa & A1 & IS & 2 & $1,2-1,13-1,4-1-1-12,18$ \\
\hline 13155 & Aug. & 2001 & $\mathrm{~F}$ & Csa & A1 & IS & 2 & $1-1,5-1,12-1-1-13,19$ \\
\hline
\end{tabular}

with an A1 (12144) and an A2 (12146) isolate retrieved from irrigation water in 2006. Otherwise, isolates that shared a multilocus genotype were found in the same year of sampling (Table 1).

No deviations from neutrality were observed in $\beta$-tubulin or TigA (Table 4). Significant and positive values for Tajima's D and $\mathrm{Fu}$ and Li's $\mathrm{D}$ and $\mathrm{F}$ indicate balancing selection or a recent population contraction for EF-1 $\alpha$, HSP90, and Ura3. Using the four-gamete test of Hudson and Kaplan (17), the minimum number of recombination events (RM) was estimated for each gene. Four of the six nuclear genes tested yielded $R M \geq 2$ (Table 4). Split networks and statistical-parsimony haplotype genealogies created for the six nuclear genes support these results (Figs. 1, 2, and 3).

NeighborNet networks of $P$. capsici haplotypes showed no distinctive groups with high bootstrap support that were consistent in all or most networks (Figs. 1 and 2). Networks overall indicated significant incompatibility within nuclear genes, and the highest amount of incompatibilities was observed in Enolase (Figs. 1 and 2). Statistical-parsimony genealogies presented a similar topology to the NeighborNet networks (Fig. 3). P. capsici haplotypes appeared to be very closely related to each other, with only one or two missing haplotypes separating sampled haplotypes. Genealogies included $P$. capsici haplotypes that were sampled in all genetic clusters, some clusters, or that were unique to a particular cluster (Table 1). Haplotypes only found in the creek, the field, or obtained from both sources were also observed (Fig. 3, Table 1). Unique and shared haplotypes were also detected for all years (Table 1). Shared haplotypes among isolate sources, years, or clusters were frequently determined to have the highest probability of being the ancestral haplotype in the statistical-parsimony genealogies (Fig. 3).

Isolates were clustered into underlying genetic groups using Bayesian clustering. Population structure analysis revealed that there were likely four genetic clusters $(\mathrm{k}=3 \mathrm{LnP}=-1759, \mathrm{k}=4$ $\mathrm{LnP}=-1669, \mathrm{k}=5 \mathrm{LnP}=-1930)$. The average proportion of ancestry (Q) assigned to individuals was highly consistent across replicate runs. Some isolates were highly admixed, and others belonged predominantly to one cluster. Clusters did not correspond directly to year of sampling, mating type, or sensitivity to mefenoxam, and all of the genetic clusters were present in all of the categories we examined (Fig. 4). Isolates were collected from infected cucumbers in 2001 and were baited from the water using cucurbit fruits in 2002, 2003, and 2006. Hence, genetic clusters do not directly correspond to the source of the isolates (field versus water) either. All of the isolates retrieved from the field and from the water were pathogenic to pepper fruit (data not shown).

Population structure was detected when data were sorted using year, mating type, and mefenoxam sensitivity as prior population information (Fig. 4). While isolates belonging to each of the four clusters were present in every category investigated, genotypes with predominant membership in certain clusters were more common in some categories than others. In 2001, isolates in cluster two were the most common, representing 53\% (8 of 15) of the isolates sampled. Only one isolate with predominant membership in cluster three was sampled in 2001. Isolates from cluster one and cluster 
four represented 27 and $13 \%$ of the total isolates sampled, respectively. In 2002, the majority of isolates $(43 \%, 10$ of 23$)$ belonged mostly to cluster one (Fig. 4); no isolates with predominant membership in cluster four were detected. Isolates mostly from cluster three $(36 \%, 9$ of 25$)$ and cluster one $(32 \%, 8$ of 25$)$ were more common in 2003, but isolates were also collected from clusters two (12\%) and four (8\%). In 2006, isolates predominantly belonging to cluster four were sampled much more frequently $(64 \%, 27$ of 42$)$ than isolates from the other three clusters $(\leq 17 \%$ each). Several isolates from each year $(0,3,3$, and 2 isolates, respectively) were evenly admixed between two or more clusters and could not be assigned predominant membership. Most of the isolates displayed some admixture (Fig. 4).

Cucumbers were grown at this farm in 2001 and 2002; during this time period, isolates from clusters one and two $(37 \%$ each, 14 of 38) were more common than isolates from clusters three $(13 \%)$ and four (5\%) (Fig. 4). When nonhost crops were grown in 2003 and 2006 (potato and unknown agronomic crop, respectively), isolates from cluster two were rare (4 of 68), but the other clusters were well represented $(\geq 15$ isolates with predominant

Table 2. Genes, primer information, source sequence used for primer design, and size of analyzed sequence for target mitochondrial and nuclear genes in Phytophthora capsici

\begin{tabular}{|c|c|c|c|c|c|c|}
\hline Target DNA & Primer & Primer sequence & $\begin{array}{l}\text { Annealing } \\
\text { temp. }\left({ }^{\circ} \mathrm{C}\right)^{\mathrm{a}}\end{array}$ & $\begin{array}{l}\text { Size of sequenced } \\
\text { fragment (bp) }\end{array}$ & $\begin{array}{l}\text { Size of analyzed } \\
\text { region (bp) }\end{array}$ & Source \\
\hline \multicolumn{7}{|l|}{ Mitochondrial } \\
\hline \multirow[t]{2}{*}{ Cox 1} & Cox1-F & 5'GGTGCACCTGATATGGCTTT3' & 60 & 425 & 355 & AY129166.1 \\
\hline & Cox1-R & 5'ACAGGATCACCTCCACCTGA3' & 60 & & & \\
\hline \multirow[t]{2}{*}{$\operatorname{Cox} 2$} & $\operatorname{Cox} 2-\mathrm{F}$ & 5'CCAGCAACTCCTGTAATGGAA3' & 60 & 540 & 365 & DQ365739.1 \\
\hline & Cox $2-\mathrm{R}$ & 5'TTGATTTAAACGGCCAGGAC3' & 59 & & & \\
\hline \multirow[t]{2}{*}{ Nad1 } & Nad1-F & 5'CAAAGAAGAAGAGGACCTAATGTTG3' & 59 & 579 & 430 & DQ361203 \\
\hline & Nad2-R & 5'TAATGCAAAACCCATTGCAG3' & 59 & & & \\
\hline \multirow[t]{2}{*}{ Nad5 } & Nad5-F & 5'GCTATGGAAGGTCCTACACCA3' & 58 & 341 & 245 & AY423326.1 \\
\hline & Nad5-R & 5'GCATGGATTACTGCACCTGA3' & 58 & & & \\
\hline \multicolumn{7}{|c|}{ รеง } \\
\hline \multirow[t]{2}{*}{$\beta$-tubulin } & BTub-F & 5'GGTCAGTGCGGTAACCAGAT3' & 60 & 597 & 505 & EF495258.1 \\
\hline & BTub-R & 5'GTACAGGGCCTCGTTATCCA3' & 59 & & & \\
\hline \multirow[t]{2}{*}{$\mathrm{EF}-1 \alpha$} & EF1A-F & 5'GACATTGCCCTGTGGAAGTT3' & 59 & 568 & 450 & EU079545.1 \\
\hline & EF1A-R & 5'CAGGCTTGATGACACCAGTC $3^{\prime}$ & 59 & & & \\
\hline \multirow[t]{2}{*}{ Enolase } & Enolase-F & 5'CGTGAAGAACGTGAACGAGA3' & 60 & 647 & 435 & EU080622.1 \\
\hline & Enolase-R & 5'CCGAGATCTTCTCCGACTCC3' & 57 & & & \\
\hline \multirow[t]{2}{*}{ HSP90 } & HSP90-F & 5'GCCGATCTCATCAACAACCT3' & 60 & 542 & 465 & EU079547.1 \\
\hline & HSP90-R & 5'CTTCTGCGAGTTCAGGTGGT3' & 60 & & & \\
\hline \multirow{2}{*}{ TigA } & TigA-F & 5'TCAАCACTGCCAAAATTCCA3' & 60 & 516 & 420 & EU080625.1 \\
\hline & TigA-R & 5'CAGCGTCAGAGGAGACCTTC $3^{\prime}$ & 60 & & & \\
\hline \multirow[t]{2}{*}{ Ura3 } & Ura3-F & 5'GGCTTTCGACCAGCTGAAT3' & 59 & 570 & 500 & EF617399.1 \\
\hline & Ura3-R & 5'AGCGTGAAGTCACCGAACTT3' & 59 & & & \\
\hline
\end{tabular}

${ }^{a}$ Calculated annealing temperature from Primer3. The actual annealing temperature used was $56^{\circ} \mathrm{C}$ for all genes.

Table 3. Polymorphism types for target mitochondrial and nuclear genes in Phytophthora capsici

\begin{tabular}{|c|c|c|c|c|c|}
\hline Target DNA & $\begin{array}{c}\text { Parsimony } \\
\text { informative sites }\end{array}$ & Synonymous changes & Replacement changes & Transitions & Transversions \\
\hline Mitochondrial $^{\mathrm{a}}$ & 0 & 0 & 0 & 0 & 0 \\
\hline \multicolumn{6}{|l|}{ Nuclear } \\
\hline$\beta$-tubulin & 7 & 6 & 1 & 6 & 1 \\
\hline EF-1 $\alpha$ & 7 & 7 & 0 & 7 & 0 \\
\hline Enolase & 11 & 8 & 3 & 10 & 1 \\
\hline HSP90 & 4 & 1 & 3 & 2 & 2 \\
\hline TigA & 4 & 4 & 0 & 4 & 0 \\
\hline Ura3 & 16 & 11 & 5 & 11 & 5 \\
\hline Subtotal & 49 & 37 & 12 & 40 & 9 \\
\hline Total & 49 & 37 & 12 & 40 & 9 \\
\hline
\end{tabular}

${ }^{a}$ Four regions of the mitochondrial genome (Cox1, Cox2, Nad1, and Nad5) were sequenced.

Table 4. Diversity estimates, neutrality tests and recombination for target mitochondrial and nuclear genes in Phytophthora capsici ${ }^{\mathrm{a}}$

\begin{tabular}{|c|c|c|c|c|c|c|c|c|c|c|c|}
\hline \multirow[b]{2}{*}{ Target DNA } & \multicolumn{6}{|c|}{ Diversity estimates } & \multicolumn{2}{|c|}{$\begin{array}{c}\text { Recombination } \\
\text { estimates }\end{array}$} & \multicolumn{3}{|c|}{ Neutrality tests } \\
\hline & $\mathrm{s}(\%)$ & $\mathbf{h}$ & Hd & $\pi$ & $\theta \mathbf{w}$ & $\mathbf{k}$ & $\mathbf{R}$ & $\mathbf{R M}$ & Tajima's D* & Fu and Li's D* & Fu and Li's F* \\
\hline Mitochondrial $^{\mathrm{b}}$ & $0(0)$ & 1 & 0 & NA & NA & NA & NA & NA & NA & NA & NA \\
\hline$\beta$-tubulin & $7(1)$ & 10 & 0.626 & 0.003 & 1.18 & 1.667 & 3.7 & 2 & 0.869 & 1.136 & 1.244 \\
\hline EF-1 $\alpha$ & $7(2)$ & 14 & 0.711 & 0.006 & 1.18 & 2.685 & 5.7 & 3 & $2.69^{*}$ & 1.136 & $2.004 *$ \\
\hline Enolase & $11(3)$ & 24 & 0.821 & 0.004 & 1.855 & 1.764 & 29.6 & 5 & -0.115 & 1.38 & 0.994 \\
\hline HSP90 & $4(1)$ & 5 & 0.091 & 0 & 0.674 & 0.092 & 0 & 0 & -1.523 & $-3.016^{*}$ & $-2.989 *$ \\
\hline TigA & $4(1)$ & 4 & 0.346 & 0.002 & 0.674 & 0.736 & 0 & 0 & 0.161 & 0.88 & 0.76 \\
\hline Ura3 & $16(3)$ & 22 & 0.787 & 0.01 & 2.697 & 4.758 & 10.4 & 6 & 1.956 & $1.605^{*}$ & $2.091 *$ \\
\hline
\end{tabular}

${ }^{a}$ s: number of polymorphisms. h: number of haplotypes. Hd: haplotype diversity. $\pi$ : nucleotide diversity. $\theta \mathrm{w}$ : Watterson's theta estimator per gene from sequence. $\mathrm{k}$ : average number of nucleotide differences. R: recombination parameter. RM: minimum number of recombination events. * $=$ significant at 0.05 .

${ }^{\mathrm{b}}$ Four regions of the mitochondrial genome (Cox1, Cox2, Nad1, and Nad5) were sequenced. 
membership in each cluster) (Fig. 4). Isolates belonging predominantly to cluster one more often possessed an A1 (66\%) than A2 (34\%) mating type. Conversely, cluster three and four isolates were more likely to be A2 (76\%) than A1 (24\%). The percentage of isolates sampled that were insensitive to mefenoxam was much greater in cluster four $(81 \%)$ and cluster three $(65 \%)$ than for isolates mostly in cluster one $(41 \%)$ and cluster two (33\%).

As a measure of genetic differentiation, pairwise $\mathrm{F}_{\mathrm{ST}}$ values were calculated for groups of isolates separated by year (2001, 2002, 2003, 2006), source (field versus water), mating type (A1 versus A2), and mefenoxam sensitivity (insensitive, intermediately sensi-
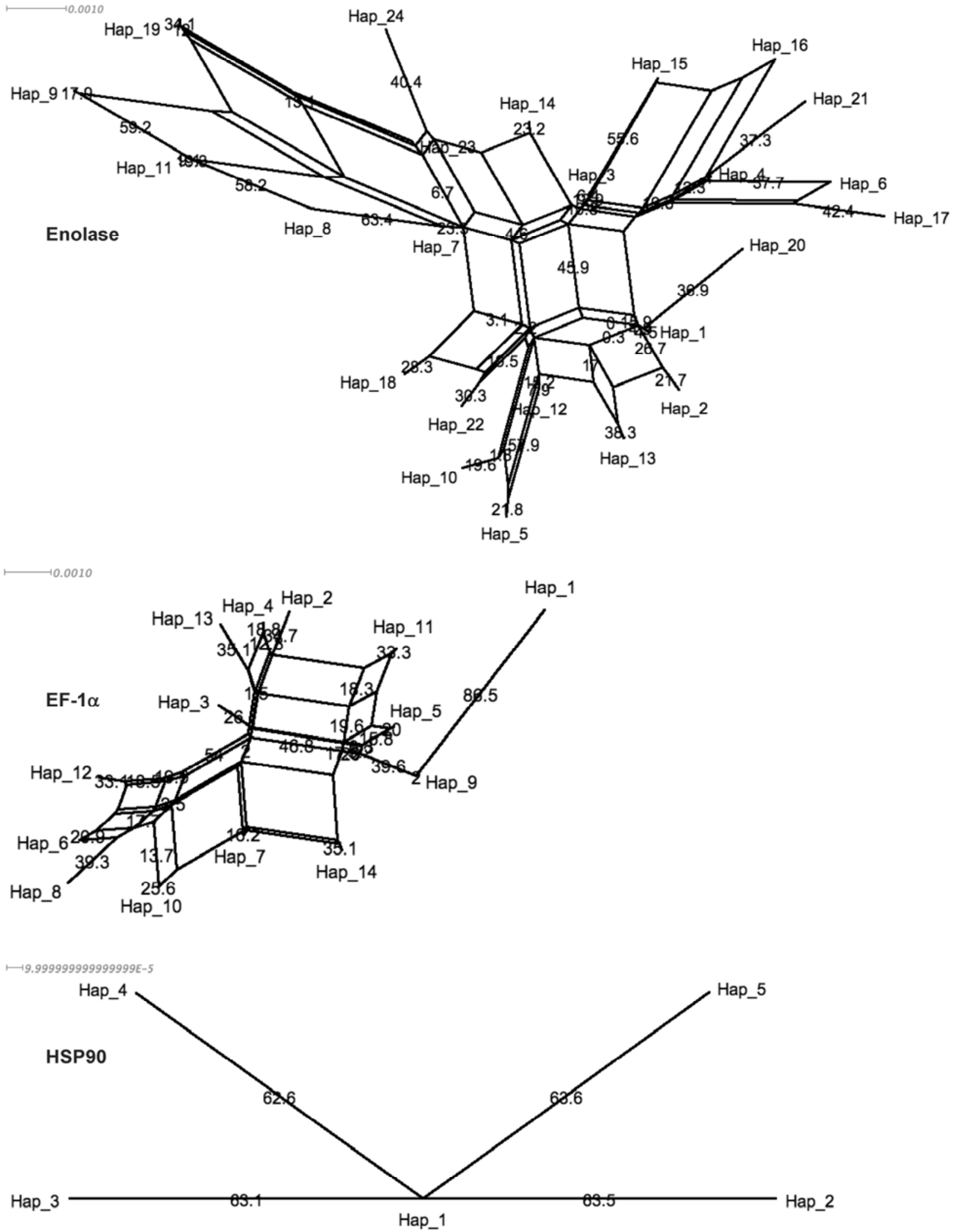

Fig. 1. Split network analysis of Phytophthora capsici nuclear genes (Enolase, EF1- $\alpha$, and HSP90). Parallel lines linking isolates represent incompatibilities. Bootstrap support for each connecting line is indicated. 
tive, and sensitive). Genetic differentiation estimates indicated low to moderate differentiation between categories, supporting moderate to high gene flow between populations (Table 5). The highest $\mathrm{F}_{\mathrm{ST}}$ values were observed when 2001 and 2002 isolates were compared to isolates collected in 2006 , and these values indicated moderate differentiation (Table 5). The highest diversity estimates for isolates grouped by years were for isolates from 2001, which indicates higher genetic diversity was found in the field samples as compared with samples baited from the water (Table 6). Diversity estimates for isolates grouped by mating type were similar, as were estimates for isolates grouped by mefenoxam sensitivity (Table 6). Shifts in phenotypic traits were observed over time, with a higher

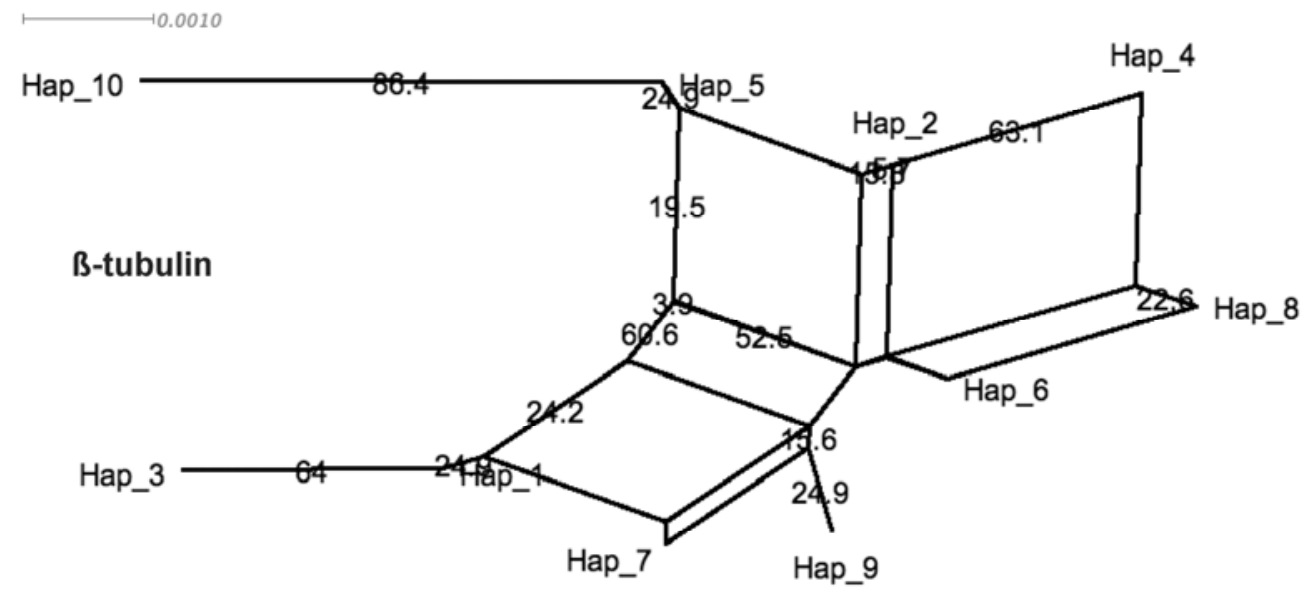

TigA
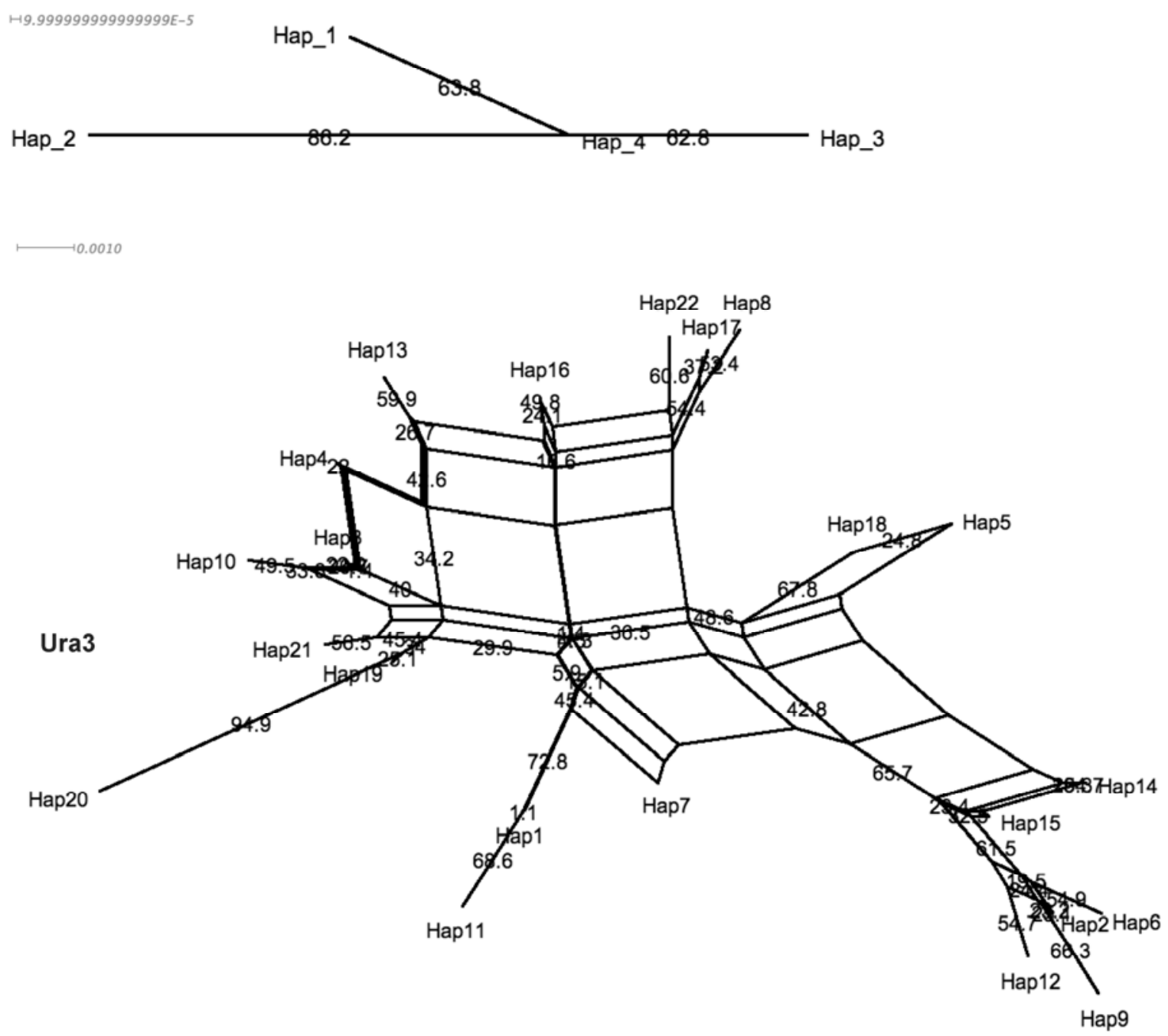

Fig. 2. Split network analysis of Phytophthora capsici nuclear genes ( $\beta$-tubulin, TigA, and Ura3). Parallel lines linking isolates represent incompatibilities. Bootstrap support for each connecting line is indicated. 
proportion of insensitive isolates in 2003 (24 of 25) and 2006 (29 of 43) than in 2001 ( 1 of 15) and 2002 (7 of 23). In addition, the mating type ratio was skewed toward A1 in 2001 (11:4), was evenly distributed in the 2002 (12:11) and 2003 (14:11) samplings, and was highly skewed toward the A2 mating type in the 2006 sample (10:33).

\section{Discussion}

This study investigated the genetic structure of $P$. capsici populations from a creek used for irrigating fields at a farm in Southwest Michigan. Four genetic clusters were detected using Bayesian clustering; these clusters did not correspond directly with the four years of sampling, isolate source, mating type, or mefenoxam sensitivity categories. While individual genetic clusters did not directly correspond with individual years, temporal changes in population structure and in frequencies of certain phenotypic traits were observed.

Differences in cluster composition and genetic diversity were observed between isolates from 2006 and from other years. In 2006, isolates from cluster four were more frequently sampled and genetic diversity was lower than in other years. $\mathrm{F}_{\mathrm{ST}}$ values also indicated low to moderate genetic differentiation between samples collected in 2006 and individuals from other years. Results from investigations with $P$. capsici in Michigan suggest that reproduction of clonal individuals within a single season is significant
$(13,26)$, which could account at least in part for the high occurrence of isolates from cluster four in 2006. The temporal shift of cluster occurrence in our sample could also be due to changes in agricultural practices in adjacent fields. The creek would receive runoff from fields on this farm upstream of the sampling site. The grower cooperator farm, from which all of the samples were collected, transitioned from cucumber production to the production of potatoes and field crops in 2003 because of severe Phytophthora disease pressure. $P$. capsici was detected in the creek even after the adjacent farmland was transitioned to potato (2003) and an unknown agronomic crop (2006). In addition, isolates were collected from this creek in 2004 and 2005, when the adjacent fields were planted to soybean and corn, respectively (9). Isolates in cluster four were relatively rare when the farm was in cucumber production in 2001 and 2002. In 2003, two isolates predominantly in cluster four were sampled. In contrast, the majority of isolates collected in 2006 were in cluster four. Selection by hosts is an important evolutionary force that stratifies plant pathogen populations $(32,50)$. Homogeneous crops grown over large areas can also result in directional selection of local pathogen populations, causing selective sweeps that will favor particular genotypes (2). When nonhost crops were grown in the field, populations that could survive in the absence of a host may have been selected for over time. The selected genotypes in the field could have infested nearby irrigation water
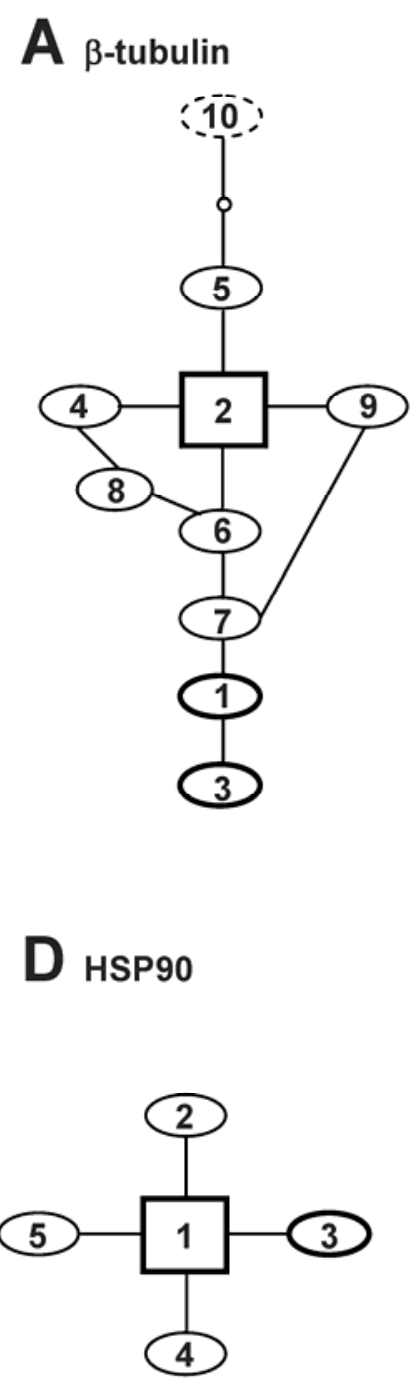
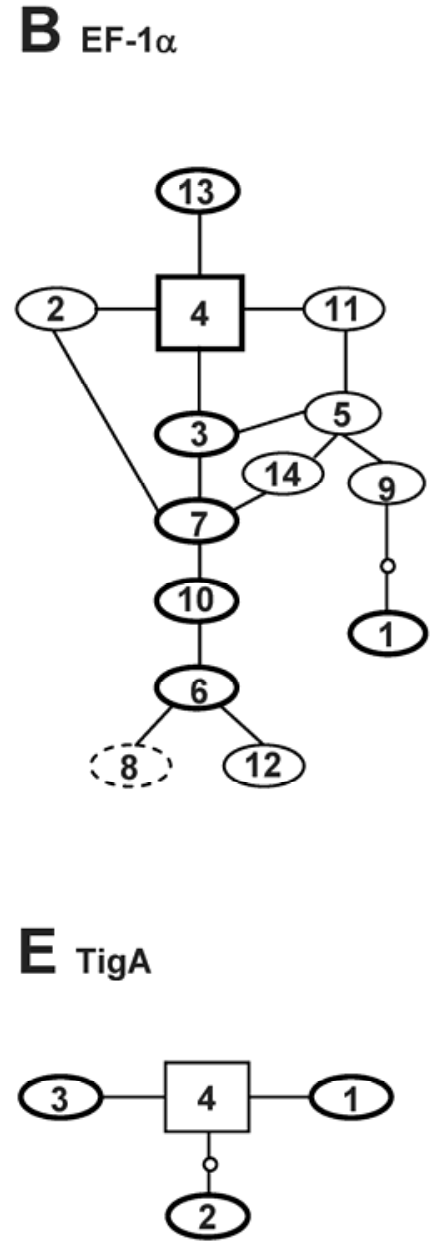
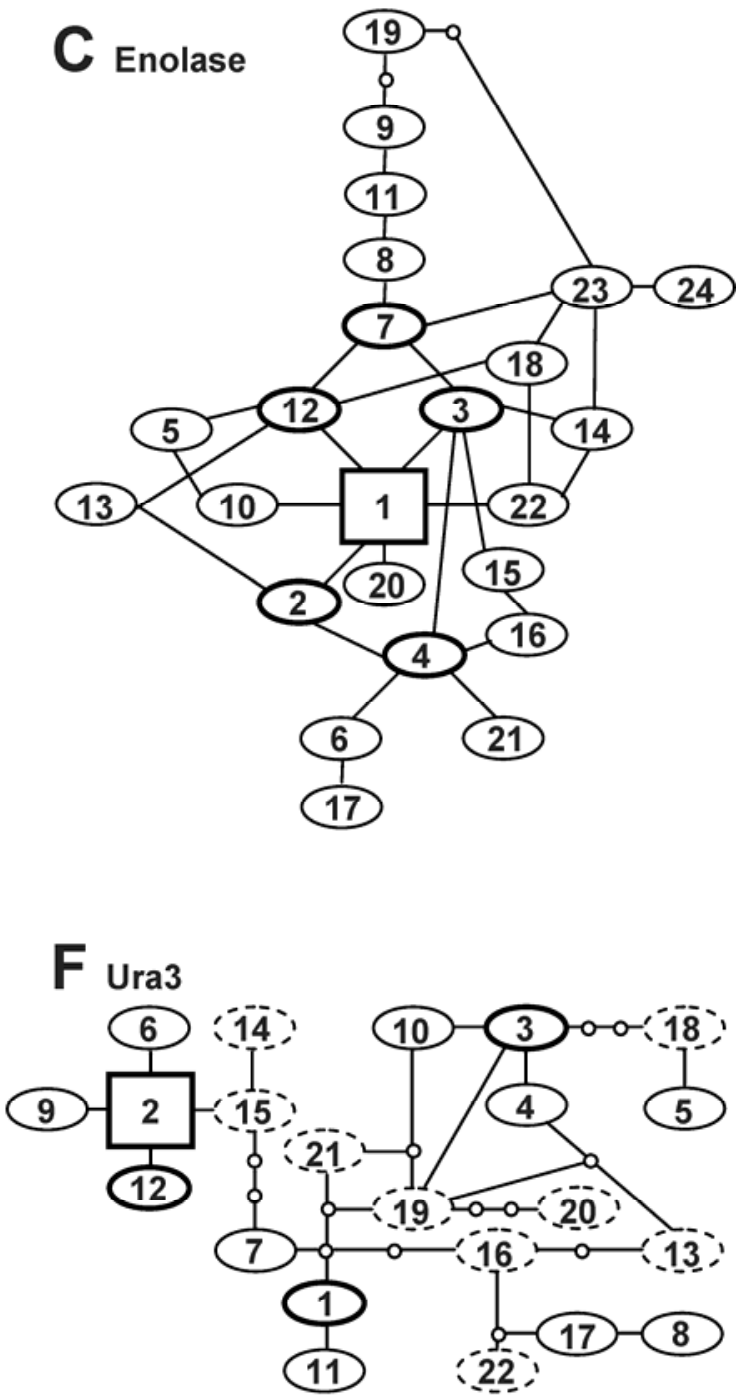

Fig. 3. Statistical-parsimony genealogies of Phytophthora capsici nuclear genes haplotypes. The lines in the network represent possible paths of evolution. The haplotype with the highest probability to be ancestral is indicated by a square; other haplotypes are indicated by ovals. Dashed ovals represent haplotypes found only in the field (2001) sampling, bolded ovals represent haplotypes found in both field and creek samplings, and unmodified ovals represent haplotypes found only in the creek. Empty dots represent missing or extant haplotypes. 
sources and resulted in a dominance of isolates from cluster four during 2006.

Selection of particular genotypes in 2006 could have also been due to pressure from fungicides applied to crops in the adjacent fields. The fungicide mefenoxam is commonly applied to protect susceptible crops from P. capsici. Mefenoxam is also applied to protect potatoes from Phytophthora infestans and soybeans from $P$. sojae (11). Nonetheless, Phytophthora populations resistant to the fungicide have been documented $(11,24,25,29,34)$. In our study, a shift in mefenoxam sensitivity appeared to occur in 2003, and more insensitive isolates were observed in 2003 and 2006 than in 2001 and 2002. Gevens et al. (9) used some of the same isolates included in our study and found a higher proportion of sensitive isolates in her 2005 sampling at this site, with $78 \%$ (18 of 23) isolates tested being fully sensitive to mefenoxam. The findings of Gevens et al. (9) do not support a cumulative selection effect of phenylamide fungicides on $P$. capsici water populations at this site. Thus, yearly changes in isolate phenotypic traits, such as mefenoxam sensitivity and mating type, appear to be occurring at this site. While Lamour and Hausbeck (26) found $\mathrm{A} 1: \mathrm{A} 2$ mating type ratios of approximately $1: 1$ and similar mefenoxam sensitivity frequencies over a 2-year time period at their field sites, we found a marked deviation from a 1:1 mating type ratio in 2001 and in 2006, when the A1 and A2 mating type, respectively, appeared more prevalent in our sampling.
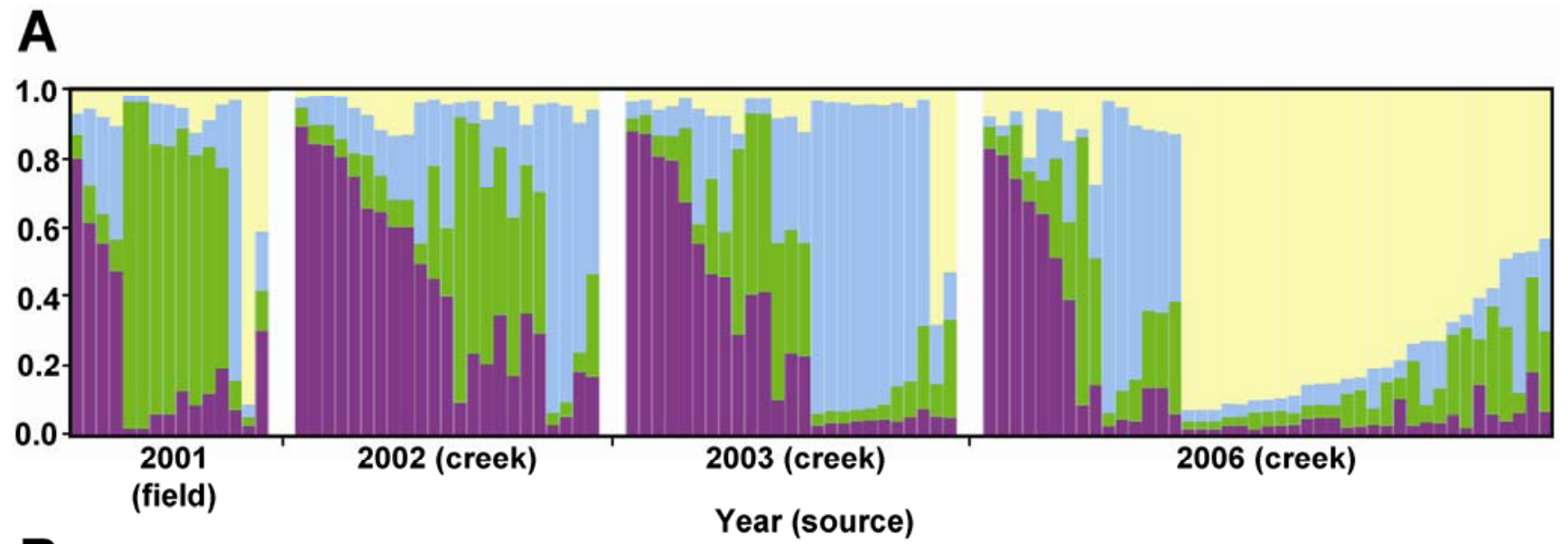

B

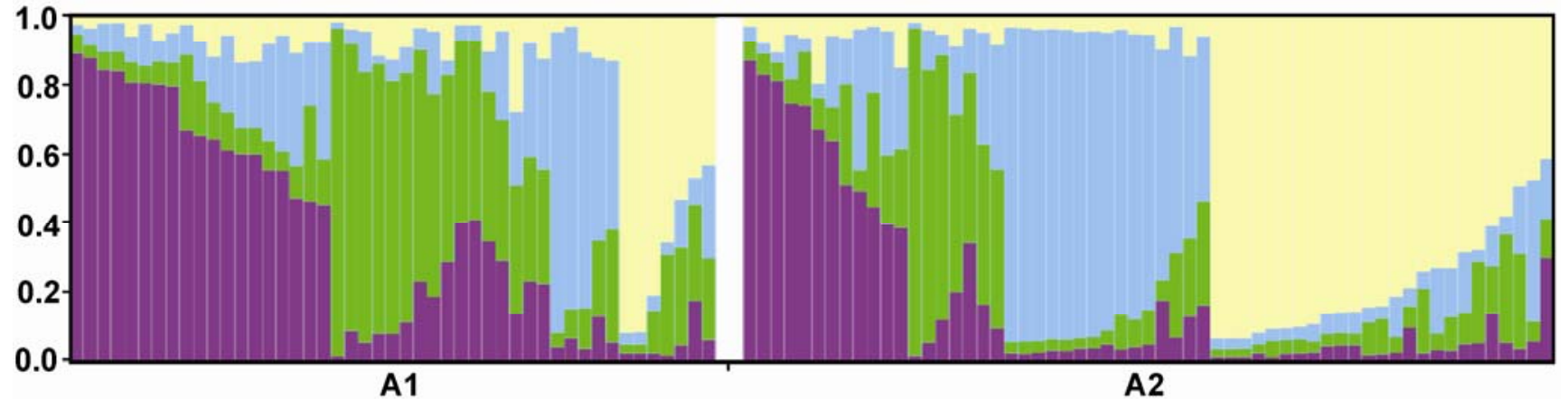

Mating Type

C

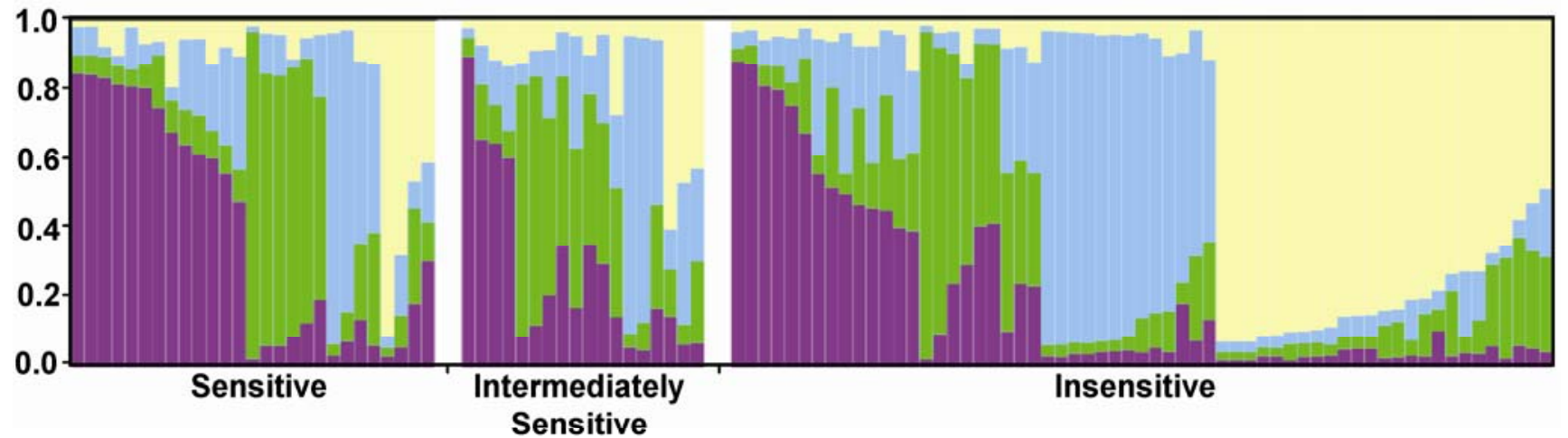

\section{Sensitivity to Mefenoxam}

Fig. 4. Estimated population structure of 106 isolates of Phytophthora capsici collected from a field and creek on a farm in Southwest Michigan. Each isolate is represented by a single vertical bar, which is divided into colored segments with lengths representing proportional estimated membership of the isolate in each of the four clusters identified by Bayesian clustering. A, B, and C display the same isolates, but are sorted by A, year, B, mating type, and C, sensitivity to the fungicide mefenoxam. Isolates from the field were collected in 2001 and isolates from the creek in 2002, 2003, and 2006. Colors correspond to clusters as follows: purple = cluster 1, green = cluster 2, blue $=$ cluster 3 , and yellow $=$ cluster 4 . 
Isolates from years 2001, 2002, and 2003 presented similar cluster composition and low genetic differentiation. This could be due to the pathogen overwintering in irrigation water or in adjacent fields and washing into the water source annually. $P$. capsici can persist in soil for several years as oospores (13), and P. capsici could be persisting in surface water irrigation source sediment as oospores also. Runoff water from infested fields can transport $P$. capsici to nearby water sources used for irrigation (13). Some isolates from the field (2001) could be persisting in soil several years as oospores and infesting the creek every year through runoff water. Our study identified haplotypes shared between the field and water isolates, and isolates sampled in different years. Moreover, shared haplotypes between field and water isolates were usually inferred as being the ancestral haplotype in our genealogical analyses and were sampled almost every year. However, the statisticalparsimony genealogies also detected haplotypes that were unique to the field or the creek, suggesting that sources other than the field could be contributing inocula to the creek. Infested irrigation water is an efficient means of spreading inocula from one geographic site to other locations using the same water system $(22,33)$. In a study conducted by Oudemans in New Jersey, P. cinnamomi was found in water upstream of any agricultural land, suggesting that this pathogen probably survives on native vegetation in areas that are sources for streams sampled in the study (33). P. capsici has a broad host range (5), it can infect diverse weeds (7), and oospores have been found in the digestive tracts of insects (13). Thus, $P$. capsici could be surviving on vegetation or insects in or nearby the creek. While it is unlikely that zoospores could overwinter in irrigation water due to a transient and thin cell wall (20), some Phytophthora spp. can survive as chlamydospores in water at room temperature for 6 to 23 years (21). Some $P$. capsici isolates form chlamydospores in liquid culture at approximately $18^{\circ} \mathrm{C}(19)$, but it is not known whether chlamydospores are formed in irrigation water or if they can function as overwintering structures. In addition, Pythium aphanidermatum hyphae in rye seed and oospores were able to survive for at least 185 days in a grower's irrigation pond in Georgia (44). If oospores or infected plant material are washing into nearby irrigation sources, $P$. capsici survival in irrigation water could be more likely. With the current data available, we cannot determine if $P$. capsici overwintered in the creek or was transported from the field or other inocula sources to the creek each year. Future studies aimed at understanding the aquatic ecology of $P$. capsici may provide further information about survival and population dynamics of this pathogen in irrigation water.

Previous studies have relied on phylogenetic methods to analyze the population structure of $P$. capsici populations in irrigation water $(9,47)$. No more than two SSR clusters were detected for $P$. capsici retrieved from a single irrigation pond in Georgia (47), and

Table 5. Genetic differentiation $\left(\mathrm{F}_{\mathrm{ST}}\right)$ estimates for Phytophthora capsici with isolates grouped in predefined categories by year, source, mating type, mefenoxam sensitivity

\begin{tabular}{lcll}
\hline Category & \multicolumn{3}{c}{$\mathbf{F}_{\mathbf{S T}^{\mathbf{a}}}{ }^{\mathbf{a}}$} \\
\hline Year & 2002 & 2003 & 2006 \\
2001 & 0.024 & 0.032 & $0.065^{*}$ \\
2002 & & 0.024 & $0.074^{*}$ \\
2003 & & & 0.037 \\
Source & Water & & \\
Field & 0.031 & & \\
Mating type & $\mathrm{A} 2$ & & \\
A1 & 0.017 & & \\
Mefenoxam sensitivity & & & \\
& Intermediately & Sensitive & \\
Insensitive & sensitive & & \\
Intermediately sensitive & 0.008 & 0.043 & \\
\hline
\end{tabular}

\footnotetext{
a Average for all nuclear genes; $*$ = significant at 0.05 .
}

${ }^{\mathrm{b}}$ Isolate sensitivity to $100 \mathrm{ppm}$ mefenoxam. Isolates were compared to an unamended control: Sensitive: $<30 \%$ relative growth; Intermediately sensitive: 30 to $90 \%$ relative growth; Insensitive: $>90 \%$ relative growth. only three amplified fragment length polymorphism (AFLP) similarity groups were detected for a group of 56 isolates retrieved from irrigation water sources in Michigan (pond, river, creek, runoff ditch) (9). Some of the isolates from 2002 and 2003 included in our study were also included in the study by Gevens et al. (9). While Gevens et al. placed these isolates in two distinct similarity groups based on AFLP markers, we found four genetic clusters using multilocus genotype data and Bayesian clustering. The sample size was similar for 2003 ( 25 versus 24 isolates), but Gevens et al. used fewer isolates for 2002 (6 versus 23 isolates), and this may account for part of the underestimation of subpopulations. In addition, the Georgia and Michigan studies both used the unweighted pair group method with arithmetic mean (UPGMA) algorithm to construct trees displaying clusters. The Bayesian clustering approach used in our study assigns individuals in the sample to one or more genetic clusters based on probability. Pritchard et al. (36) suggested that the model-based approach implemented in Structure is better than distance-based methods, such as UPGMA clustering, for fine statistical inference. Furthermore, while recombination may result in a poorly or unreliably resolved tree when using traditional phylogenetic approaches $(35,43)$, Bayesian clustering may be used for highly recombining species (6). Recombination is common in $P$. capsici populations according to previous studies $(23,24,26)$ and to recombination estimates, statistical-parsimony genealogies, and NeighborNet networks generated in this study. Of course, our sampling represents the isolates that could be retrieved from the water using cucurbit baits and that survived long-term storage. We could be underestimating apparent structure in the creek due to the limitations in our collection of isolates or due to fact that only six polymorphic loci were used in this study (42). However, this study does show the utility of Bayesian clustering for population studies in $P$. capsici.

All of the isolates baited from the water in 2002, 2003, and 2006 were pathogenic to wounded pepper fruits, as were isolates collected from diseased cucumbers in the field in 2001. If water from this creek is used for irrigation of planted susceptible crops in adjacent fields, losses due to $P$. capsici will likely occur. If infested irrigation water cannot be avoided or if using recirculating nutrient solutions in the greenhouse, pathogen detection and quantification in water is crucial to develop successful disease management programs for vegetable crops susceptible to Phytophthora spp. (14). Molecular methods can detect as few as 20 to 300 Phytophthora spp. zoospores per liter in infested irrigation water

Table 6. Number of isolates for Phytophthora capsici and diversity estimates with isolates grouped in predefined categories

\begin{tabular}{lrcccc}
\hline & \multicolumn{5}{c}{ Diversity estimates $^{\mathbf{a}}$} \\
\cline { 2 - 6 } Category & $\mathbf{n}$ & Hd & $\boldsymbol{\pi}$ & $\boldsymbol{\theta} \mathbf{w}$ & $\mathbf{k}$ \\
\hline Year & & & & & \\
2001 & 15 & 0.611 & 0.004 & 1.515 & 2.104 \\
2002 & 23 & 0.539 & 0.004 & 1.252 & 1.823 \\
2003 & 25 & 0.527 & 0.004 & 1.34 & 1.833 \\
2006 & 43 & 0.495 & 0.004 & 1.26 & 1.838 \\
Source & & & & & \\
$\quad$ Field & 15 & 0.611 & 0.004 & 1.515 & 2.104 \\
$\quad$ Water & 91 & 0.551 & 0.004 & 1.298 & 1.898 \\
Mating type & & & & & \\
$\quad$ A1 & 47 & 0.566 & 0.004 & 1.531 & 1.929 \\
$\quad$ A2 & 59 & 0.543 & 0.004 & 1.609 & 1.904 \\
$\quad \begin{array}{l}\text { Mefenoxam sensitivity } \\
\text { Insensitive }\end{array}$ & 61 & 0.539 & 0.004 & 1.519 & 1.884 \\
$\quad$ Intermediately & 18 & 0.56 & 0.004 & 1.367 & 1.885 \\
$\quad$ sensitive & & & & & \\
Sensitive & 27 & 0.596 & 0.004 & 1.5 & 1.985 \\
\hline
\end{tabular}

${ }^{a}$ Average for all nuclear genes, $n$ : number of isolates, Hd: haplotype diversity, $\pi$ : nucleotide diversity, $\theta \mathrm{w}$ : Watterson's theta estimator per gene from sequence, and $\mathrm{k}$ : average number of nucleotide differences.

${ }^{b}$ Isolate sensitivity to $100 \mathrm{ppm}$ mefenoxam. Isolates were compared to an unamended control: Sensitive: $<30 \%$ relative growth; Intermediately sensitive: 30 to $90 \%$ relative growth; Insensitive: $>90 \%$ relative growth 
$(22,51)$. Molecular diagnostics reduces the time, training, and labor required to culture and identify $P$. capsici by traditional microscopy methods, which may delay disease-management recommendations. Methods based on PCR can be used to survey and quantify faster and more accurately the occurrence and distribution of $P$. capsici populations in infested water sources $(22,51)$. Knowledge of the genetic variation of $P$. capsici found in irrigation water is required to develop species-specific molecular diagnostic tools that allow for efficient monitoring of this pathogen in water sources. Intra-specific data from Phytophthora spp. from irrigation water are commonly genetically characterized by using markers based on the internal transcribed spacer (ITS); however, such markers are not always useful for species differentiation (49). Using several nuclear genes may be necessary to reliably differentiate $P$. capsici from other closely related species such as $P$. tropicalis that can also be found in irrigation water sources $(14,16)$. Our analyses provide information about the genetic variation of $P$. capsici in Michigan irrigation water. In addition, the gene markers and methods used in our study can be used to develop species-specific molecular diagnostics assays since they are able to differentiate $P$. capsici from $P$. tropicalis (38).

$P$. capsici has been previously found in irrigation water sources in Michigan and is thought to be a source of initial inocula for some fields (9). P. capsici can continue to contaminate irrigation water sources even after the adjacent fields have been transitioned to nonhost crops for multiple years. Low genetic differentiation was observed between isolates retrieved from the field and the creek water. Four genetic clusters were detected in our sampling and were present each year we sampled. Temporal changes in frequency were observed for isolate genetic cluster membership and for phenotypic isolate characteristics such as mating type and mefenoxam sensitivity between years. Since irrigation water can remain infested or become reinfested annually with $P$. capsici for years after the adjacent fields are transitioned to nonsusceptible crops, growers are advised to avoid potentially infested irrigation water even after rotating to nonhost crops for several years.

\section{Acknowledgments}

We thank all the members of the Hausbeck lab for their valuable suggestions and help. We thank J. J. Morrice for providing the program PST to generate population structure figures. We also thank K. Heslip, A. Lebeis, M. Mercier, H. Gutting, J. Olsen, H. Sweet, R. Heslip, and M. Wood for technical assistance, and A. Gevens for careful collection and cataloging of isolates during her time in the Hausbeck Lab. This material is based upon work supported by the Michigan State University Michigan Agriculture Experiment Station Hatch Project 01966, the United States Department of Agriculture (USDA) Cooperative State Research, Education and Extension Service (CSREES), and the USDA-CSREES Special Research Grant under Awards 2006-34572-16902 and 2008-3457216902.

\section{Literature Cited}

1. Altschul, S. F., Madden, T. L., Schaffer, A. A., Zhang, J., Zhang, Z., Miller, W., and Lipman, D. J. 1997. Gapped BLAST and PSI-BLAST: A new generation of protein database search programs. Nucleic Acids Res. 25:3389-3402

2. Barrett, L. G., Thrall, P. H., Burdon, J. J., and Linde, C. C. 2008. Life history determines genetic structure and evolutionary potential of hostparasite interactions. Trends Ecol. Evol. 23:678-685.

3. Calvo-Bado, L. A., Pettitt, T. R., Parsons, N., Petch, G. M., Morgan, J. A. W., and Whipps, J. M. 2003. Spatial and temporal analysis of the microbial community in slow sand filters used for treating horticultural irrigation water. Appl. Environ. Microbiol. 69:2116-2125.

4. Clement, M., Posada, D., and Crandall, K. 2000. TCS: A computer program to estimate gene genealogies. Mol. Ecol. 9:1657-1660.

5. Erwin, D. C., and Ribeiro, O. K. 1996. Phytophthora Diseases Worldwide. American Phytopathological Society, St. Paul, MN.

6. Falush, D., Stephens, M., and Pritchard, J. K. 2003. Inference of population structure using multilocus genotype data: Linked loci and correlated allele frequencies. Genetics 164:1567-1587.

7. French-Monar, R. D., Jones, J. B., and Roberts, P. D. 2006. Characterization of Phytophthora capsici associated with roots of weeds on Florida vegetable farms. Plant Dis. 90:345-350.

8. Garibaldi, A., Minuto, A., Grasso, V., and Gullino, M. L. 2003. Application of selected antagonistic strains against Phytophthora cryptogea on gerbera in closed soilless systems with disinfection by slow sand filtration. Crop Prot. 22:1053-1061.
9. Gevens, A. J., Donahoo, R. S., Lamour, K. H., and Hausbeck, M. K. 2007. Characterization of Phytophthora capsici from Michigan surface irrigation water. Phytopathology 97:421-428.

10. Ghimire, S. R., Richardson, P. A., Moorman, G. W., Lea-Cox, J. D., Ross, D. S., and Hong, C. X. 2009. An in-situ baiting bioassay for detecting Phytophthora species in irrigation runoff containment basins. Plant Pathol. 58:577-583.

11. Gisi, U., and Cohen, Y. 1996. Resistance to phenylamide fungicides: A case study with Phytophthora infestans involving mating type and race structure. Annu. Rev. Phytopathol. 34:549-572.

12. Granke, L. L., and Hausbeck, M. K. 2010. Effects of temperature, concentration, age, and algaecides on Phytophthora capsici zoospore infectivity. Plant Dis. 94:54-60.

13. Hausbeck, M. K., and Lamour, K. H. 2004. Phytophthora capsici on vegetable crops: Research progress and management challenges. Plant Dis. 88:1292-1303.

14. Hong, C., Richardson, P. A., and Kong, P. 2008. Pathogenicity to ornamental plants of some existing species and new taxa of Phytophthora from irrigation water. Plant Dis. 92:1201-1207.

15. Hong, C. X., Richardson, R. P., and Bush, E. A. 2003. Efficacy of chlorine on multiple species of Phytophthora in recycled nursery irrigation water. Plant Dis. 87:1183-1189.

16. Hong, C. X., Richardson, P. A., Kong, P., Jeffers, S. N., and Oak, S. W. 2006. Phytophthora tropicalis isolated from diseased leaves on Pieris japonica and Rhododendron catawbiense and found in irrigation water and soil in Virginia. Plant Dis. 90:525.

17. Hudson, R. R., and Kaplan, N. L. 1985. Statistical properties of the number of recombination events in the history of a sample of DNA sequences. Genetics 111:147-164.

18. Huson, D. H., and Bryant, D. 2006. Application of phylogenetic networks in evolutionary studies. Mol. Biol. Evol. 23:254-267.

19. Islam, S. Z., Babadoost, M., Lambert, K. N., Ndeme, A., and Fouly, H. M. 2004. Characterization of Phytophthora capsici isolates from processing pumpkin in Illinois. Plant Dis. 89:191-197.

20. Jeger, M. J., and Pautasso, M. 2008. Comparative epidemiology of zoosporic plant pathogens. Eur. J. Plant Pathol. 122:111-126.

21. Ko, W. H. 2003. Long-term storage and survival structure of three species of Phytophthora in water. J. Gen. Plant Pathol. 69:186-188.

22. Kong, P., Hong, C., Jeffers, S. N., and Richardson, P. A. 2003. A speciesspecific polymerase chain reaction assay for rapid detection of Phytophthora nicotianae in irrigation water. Phytopathology 93:822-831.

23. Lamour, K. 2009. Phytophthora capsici: Sex, selection, and the wealth of variation. In: Oomycete Genetics and Genomics: Diversity, Interactions, and Research Tools. K. Lamour and S. Kamoun, eds. John Wiley \& Sons, Hoboken, NJ.

24. Lamour, K. H., and Hausbeck, M. K. 2000. Mefenoxam insensitivity and the sexual stage of Phytophthora capsici in Michigan cucurbit fields. Phytopathology 90:396-400.

25. Lamour, K. H., and Hausbeck, M. K. 2001. The dynamics of mefenoxam insensitivity in a recombining population of Phytophthora capsici characterized with amplified fragment length polymorphism markers. Phytopathology 91:553-557.

26. Lamour, K. H., and Hausbeck, M. K. 2001. Investigating the spatiotemporal genetic structure of Phytophthora capsici in Michigan. Phytopathology 91:973-980.

27. Lamour, K. H., and Hausbeck, M. K. 2002. The spatiotemporal genetic structure of Phytophthora capsici in Michigan and implications for disease management. Phytopathology 92:681-684.

28. Lamour, K. H., and Hausbeck, M. K. 2003. Effect of crop rotation on the survival of Phytophthora capsici in Michigan. Plant Dis. 87:841-845.

29. Lamour, K. H., and Hausbeck, M. K. 2003. Susceptibility of mefenoxamtreated cucurbits to isolates of Phytophthora capsici sensitive and insensitive to mefenoxam. Plant Dis. 87:920-922.

30. Librado, P., and Rozas, J. 2009. DnaSP v5: A software for comprehensive analysis of DNA polymorphism data. Bioinformatics 25:1451-1452.

31. Maddison, W. P., and Maddison, D. R. 2002. MacClade: Analysis of phylogeny and character evolution. 4.06 OSX, Sunderland, MA.

32. Montarry, J., Corbiere, R., Lesueur, S., Glais, I., and Andrivon, D. 2006. Does selection by resistant hosts trigger local adaptation in plant-pathogen systems? J. Evol. Biol. 19:522-531.

33. Oudemans, P. V. 1999. Phytophthora species associated with cranberry root rot and surface irrigation water in New Jersey. Plant Dis. 83:251-258.

34. Parra, G., and Ristaino, J. B. 2001. Resistance to mefenoxam and metalaxyl among field isolates of Phytopththora capsici causing Phytophthora blight of bell pepper. Plant Dis. 85:1069-1075.

35. Posada, D., and Crandall, K. A. 2002. The effect of recombination on the accuracy of phylogeny estimation. J. Mol. Evol. 54:396-402.

36. Pritchard, J. K., Stephens, M., and Donnelly, P. 2000. Inference of population structure using multilocus genotypic data. Genetics 155:945959.

37. Quesada-Ocampo, L. M., Fulbright, D. W., and Hausbeck, M. K. 2009 Susceptibility of Fraser fir to Phytophthora capsici. Plant Dis. 93:135-141.

38. Quesada-Ocampo, L. M., Granke, L. L., Mercier, M. R., Olsen, J., and Hausbeck, M. K. 2011. Investigating the genetic structure of Phytophthora 
capsici populations. Phytopathology 101:1061-1073.

39. R-Development-Core-Team. 2008. R: A language and environment for statistical computing. R Foundation for Statistical Computing, Vienna, Austria.

40. Ristaino, J. B., Larkin, R. P., and Campbell, C. L. 1993. Spatial and temporal dynamics of Phytophthora epidemics in commercial bell pepper fields. Phytopathology 83:1312-1320.

41. Roberts, P. D., French-Monar, R. D., Hoffine, M. S., Seijo, T. E., and McGovern, R. J. 2005. Survival and recovery of Phytophthora capsici and oomycetes in tailwater and soil from vegetable fields in Florida. Ann. Appl. Biol. 146:351-359.

42. Rosenberg, N. A., Mahajan, S., Ramachandran, S., Zhao, C., Pritchard, J. K., and Feldman, M. W. 2005. Clines, clusters, and the effect of study design on the inference of human population structure. PLoS Genet 1:e70.

43. Schierup, M. H., and Hein, J. 2000. Consequences of recombination on traditional phylogenetic analysis. Genetics 156:879-891.

44. Shokes, F. M., and McCarter, S. M. 1979. Occurrence, dissemination, and survival of plant pathogens in surface irrigation ponds in southern Georgia. Phytopathology 69:510-516.

45. Stanghellini, M. E., Kim, D. H., Rasmussen, S. L., and Rorabough, P. A. 1996. Control of root rot of peppers caused by Phytophthora capsici with a nonionic surfactant. Plant Dis. 80:1113-1116.

46. Stephens, M., Smith, N. J., and Donnelly, P. 2001. A new statistical method for haplotype reconstruction from population data. Am. J. Hum. Genet. 68:978-989.

47. Wang, Z., Langston, D. B., Csinos, A. S., Gitaitis, R. D., Walcott, R. R., and $\mathrm{Ji}$, P. 2009. Development of an improved isolation approach and simple sequence repeat markers to characterize Phytophthora capsici populations in irrigation ponds in southern Georgia. Appl. Environ. Microbiol. 75:54675473.

48. Waterhouse, G. M. 1963. Key to the Species of Phytophthora de Bary. Commonwealth Mycological Society, Kew, Surrey, UK.

49. Yamak, F., Peever, T. L., Grove, G. G., and Boal, R. J. 2002. Occurrence and identification of Phytophthora spp. pathogenic to pear fruit in irrigation water in the Wenatchee River Valley of Washington State. Phytopathology 92:1210-1217.

50. Zhan, J., Mundt, C. C., Hoffer, M. E., and McDonald, B. A. 2002. Loca adaptation and effect of host genotype on the rate of pathogen evolution: An experimental test in a plant pathosystem. J. Evol. Biol. 15:634-647.

51. Zhang, Z. G., Li, Y. Q., Fan, H., Wang, Y. C., and Zheng, X. B. 2006 Molecular detection of Phytophthora capsici in infected plant tissues, soil and water. Plant Pathol. 55:770-775. 